\title{
An implementation of the general number field sieve
}

\author{
J. Buchmann J. Loho J. Zayer \\ Extended abstract \\ Fachbereich Informatik \\ Universität des Saarlandes \\ 66041 Saarbrücken \\ Germany
}

\begin{abstract}
It was shown in [2] that under reasonable assumptions the general number field sieve (GNFS) is the asymptotically fastest known factoring algorithm. It is, however, not known how this algorithm behaves in practice. In this report we describe practical experience with our implementation of the GNFS whose first version was completed in January 1993 at the Department of Computer Science at the Universität des Saarlandes.
\end{abstract}

\section{Introduction}

Factoring rational integers into primes is one of the most important and most difficult problems of computational number theory. It was shown in [2] that under reasonable assumptions the general number field sieve (GNFS) is the asymptotically fastest known factoring algorithm. It is, however, not known how this algorithm behaves in practice. In this report we describe practical experience with the first version of our implementation of the GNFS. For our implementation we used the methods described in [2], [3], and [7]. In the course of the implementation we have found several improvements which we will describe in the full version of this paper. In this extendend abstract we restrict ourselves to the presentation of a brief sketch of the algorithm and the numerical results.

\section{The GNFS}

Let $n \in \mathbb{N}$. If one can find two integers $x$ and $y$ with

$$
x^{2} \equiv y^{2} \text { modulo } n
$$

and $x \not \equiv \pm y$ modulo $n$, then $\operatorname{gcd}(x-y, n)$ is a non trivial divisor of $n$. Like many other factoring algorithms the GNFS factors $n$ by producing such a pair $x, y$. This is done in the following way: Let $f(x)=f_{0}+f_{1} \cdot x+\ldots+f_{d-1} \cdot x^{d-1}+x^{d} \in \mathbb{Z}[x]$ be an irreducible polynomial for which there exits $m \in \mathbb{Z}$ with $f(m) \equiv 0$ modulo 
$n$. Let $\rho$ be a zero of $f(x)$. The algorithm determines a non-empty set $S$ of pairs $(a, b)$ of relatively prime integers with the following properties

$$
\begin{aligned}
& X=\prod_{(a, b) \in S}(a+b m)=x^{2} \text { with } x \in \mathbb{Z} \\
& \gamma=\prod_{(a, b) \in S}(a+b \rho)=\delta^{2} \text { with } \delta \in \mathbb{Z}[\rho]
\end{aligned}
$$

The map $\varphi: \mathbb{Z}[\rho] \rightarrow \mathbb{Z} / n \mathbb{Z}, \rho \mapsto m \bmod n$ is a ring homomorphism. Therefore we have $x^{2} \equiv \varphi\left(\delta^{2}\right) \equiv \varphi(\delta)^{2} \bmod n$. If we set $y=\varphi(\delta)$ then we have found a congruence of the form (1) which with high probability yields a factorization of $n$.

The algorithm can thus be divided into three parts: determining the polynomial, finding the squares and extracting the square roots. In the remaining sections we describe our implementation of those parts and we give numerical examples. For background and details we refer to [2], [3] and [7].

\section{Determining the polynomial}

The first step of GNFS is to find an irreducible polynomial $f(x) \in \mathbb{Z}[x]$ of degree $d$ and a rational integer $m$, such that $f(m) \equiv 0 \bmod n$. For $n \leq 10^{60}$ we use $d=3$ and for $10^{60}<n<10^{180}$ we use $d=5$. We choose $i \in \mathbb{Z}$ such that for $m=\left\lfloor n^{\frac{1}{d}}\right\rfloor+i$ there is an expansion $n=m^{d}+f_{d-1} m^{d-1}+\ldots+f_{1} m+f_{0}$ with $-m / 2 \leq f_{j}<m / 2$. We determine that expansion and we set $f(x)=$ $x^{d}+f_{d-1} x^{d-1}+\ldots+f_{1} x+f_{0}$. There are various ways of modifying $f$. We can, for example, replace $f$ by $f+\sum_{j=1}^{d-1} c_{j}\left(x^{j}-m x^{j-1}\right)$. It is still an open question how an optimal polynomial $f$ can be found. We intend to use our implementation of the GNFS to study this question in detail. A few remarkable experimental results can be found in section 6 .

\section{Finding the squares}

To find the set $S$ of coprime pairs $(a, b) \in \mathbb{Z}^{2}$ satisfying (2) and (3) we use the standard sieve which is described in [2] or the lattice sieve which was suggested in [7].

In both algorithms we must choose two factor bases. The rational factor base $F_{R}$ is the set of all rational primes below some bound $s_{R} \in \mathbb{R}_{>0}$. The algebraic factor base is the set $F_{A}$ of all degree one prime ideals of $\mathbb{Z}[\rho]$ of norm below $s_{A} \in \mathbb{R}_{>0}$. The values for $s_{R}$ and $s_{A}$ are chosen according to experimental experience. Each prime in $F_{A}$ is represented by a pair $\left(p, c_{p}\right)$ where $c_{p}$ is a zero of $f$ modulo $p$. We also need large prime bounds $L_{R}$ and $L_{A}$ which are roughly $100 \cdot s_{R}$ or $100 \cdot s_{A}$, respectively. 
To apply the standard sieve, we fix bounds $A, B \in \mathbb{Z}_{>0}$ on $a$ and $b$, respectively. Again those values are chosen according to experimental experience. For each $b \in\{1,2, \ldots, B\}$ we determine all $a$ with $-A<a<A$ such that $\operatorname{gcd}(a, b)=1$, all of the prime factors of $a+b m$ except for at most one factor $l_{R}(a, b)$ belong to $F_{R}$ and all of the prime ideal factors of $(a+b \rho) \mathbb{Z}[\rho]$ except for at most two factors $l_{A, 1}(a, b)$ and $l_{A, 2}(a, b)$ belong to $F_{A}$. Also, the extra rational prime factors are called large rational primes and they must be below $L_{R}$. Analogously, the extra algebraic prime factors are called large algebraic primes and their norms must be below $L_{A}$. Any such pair $(a, b)$ is called a good pair. We say that a good pair without large primes is of type fff, if there is a large rational prime it is of type $p f f$. The definition of the types fpf, fpp, $p p f$ and $p p p$ is analogous. For a more detailed description of the sieve algorithm see [2].

To use the lattice sieve we divide the factor bases into two parts. The set $F_{r, s}$ of small rational primes contains all elements of $F_{R}$ no larger than $s_{R} / t$ where $t$ may be chosen between 2 and 10 . The set $F_{r, m}$ of medium primes is the complement of $F_{r, s}$ in $F_{R}$. For $q \in F_{r, m}$ the set $L R_{q}=\{(a, b): q \mid a+b m\}$ is a two dimensional lattice in $\mathbb{Z}^{2}$. If $(\underline{u}, \underline{v})$ is a basis of $L R_{q}$ then one can find good pairs $(a, b)$ whose small primes are bounded by $q$ by inspecting the vectors $c \underline{u}+d \underline{v}$ for $c, d \in \mathbb{Z},-C<c<C, 0<d<D$ where $C \in \mathbb{R}_{>0}$ and $D \in \mathbb{R}_{>0}$ are chosen according to experimental experience. For any fixed $d$ this can be done by a sieving procedure which is described in [7]. In this procedure we take advantage of the following fact: For $p \geq 2 C$ and $d \in\{1, \ldots, D\}$ there is exactly one $c_{d}$ such that $p$ is a divisor of $a+b m$ for $(a, b)=c_{d} \underline{u}+d \underline{w}$ and $-p / 2 \leq c_{d}<p / 2$. Since $c_{d}=c_{d-1}+c_{1} \bmod p$ those numbers can be very easily computed. It is even possible to determine the interesting values of $c_{d}$ for which $-C<c_{d}<C$ immediately. This leads to a significant speed up of the lattice sieve. A similiar trick can be applied to find $a+b \rho$ which factors up to large primes over $F_{A}$.

Once sufficiently many good pairs are found, we determine for each good pair $(a, b)$ the decompositions $a+b m=l_{R}(a, b) \cdot \prod_{p \in F_{R}} p^{e_{p}(a, b)}$ and $(a+b \rho) \mathbb{Z}[\rho]=$ $l_{A, 1}(a, b) \cdot l_{A, 2}(a, b) \cdot \prod_{P \in F_{A}} P^{e_{P}(a, b)}$, where $l_{R}(a, b), l_{A, 1}(a, b)$ and $l_{A, 2}(a, b)$ also may be 1 . We also determine a small set $F_{Q}$ of degree one prime ideals of $\mathbb{Z}[\rho]$ of norms bigger than $L_{A}$ and for each $Q \in F_{Q}$ we set $e_{Q}(a, b)=0$ if $a+b \rho$ is a square in $\mathbb{Z}[\rho] / Q$ and $e_{Q}(a, b)=1$ otherwise. The large primes are handled by constructing cycles as discribed in [1] and [6]. By calculating a non trivial linear dependency among the vectors $\left(\left(e_{p}(a, b)\right)_{p \in F_{R}}\left(e_{P}(a, b)\right)_{P \in F_{A}}\left(e_{Q}(a, b)\right)_{Q \in F_{Q}}\right)$ over $\mathbb{F}_{2}$ we determine the subset $S$ of the set of all pairs $(a, b)$ that we are looking for. As noted in [2] it may be necessary to replace $\gamma$ in (3) by $\left(f^{\prime}(\rho)\right)^{2} \gamma$ to guarantee that the square belongs to $\mathbb{Z}[\rho]$ rather than to the maximal order of the field $\mathbb{Q}[\rho]$.

\section{Finding the square roots}

Suppose we have found the set $S$ of coprime pairs $(a, b) \in \mathbb{Z}^{2}$ satisfying (2) and (3). Let $X=\prod_{(a, b) \in S} a+b m$ and let $\gamma=\left(f^{\prime}(\rho)\right)^{2} \prod_{(a, b) \in S} a+b \rho$. Extracting the 
square root $x$ of $X$ is very simple since we know the prime factorization of $X$. Computing the square root $\delta$ of $\gamma$ is, however, quite difficult since the coefficients in the representation $\delta=\delta_{0}+\delta_{1} \cdot \rho+\ldots+\delta_{d-1} \cdot \rho^{d-1}$ may be very large. In our implementation we use the method of Couveignes [3]. He suggests to determine a set $I$ of prime numbers which are inert in $\mathbb{Z}[\rho]$ and for each $p \in I$ to compute $\delta_{p}$ such that $\delta_{p}^{2} \equiv \gamma \bmod p$. This can easily be effected by applying a variant of Shanks' RESSOL algorithm [8]. Since we want to apply Chinese remaindering we must determine the image of the same square root for every $p \in I$. Using Newton iteration one can lift any $\delta_{p}$ to a number $\delta_{p^{2^{k}}}$ such that $\gamma \equiv \delta_{p^{j^{k}}} \bmod p^{2^{k}}$ where the exponent $k$ is chosen according to experimental experience. Chinese remaindering yields $y=\varphi(\delta)$.

The square $\gamma$ can be reduced in size by dividing it by some $(a+b \rho)^{2}$, where $(a, b)$ is a good pair without large primes on the algebraic side. Whether $\gamma$ is divisible by such a square can be easily checked by inspecting the vectors $\left(\left(e_{P}(a, b)\right)_{P \in F_{A}}\right)$ and $\left(\left(e_{P}(\gamma)\right)_{P \in F_{a}}\right)$. The following table shows the effect of this reduction when used in the factorization of the third number number in section 7 .

\begin{tabular}{|c|c|c|c|c|c|}
\hline$|S|$ & $\begin{array}{c}\#\left((a+b \rho)^{2}\right) \\
\text { reduced }\end{array}$ & $\begin{array}{c}|I|=\text { \# of } \\
\text { inert primes }\end{array}$ & $\begin{array}{c}\text { max. exp. } \\
2^{k}\end{array}$ & $\begin{array}{c}\text { maximal \# of } \\
\text { digits of } \delta_{j}\end{array}$ & $\begin{array}{c}\text { running time } \\
\text { in mips h }\end{array}$ \\
\hline 25022 & 0 & 115 & 256 & 133777 & 62.82 \\
\hline 25022 & 7398 & 60 & 256 & 69120 & 41.01 \\
\hline
\end{tabular}

\section{Quality of the polynomials}

The least well understood part in the GNFS is how to find the best polynomial $f$. In this section we illustrate that the algorithm behaves quite differently for different choices of polynomials. Let $n=68094773835969194533114212277$. Except for $m$ all the parameters were chosen identically as described in the next section. The next two tables show how different polynomials yield a different number of good pairs. For the first table we used the m-adic expansion as described in section 3 to find the polynomial, where $m=\left\lfloor n^{1 / 3}\right\rfloor+i$. From a bigger experiment we present the most interesting results.

\footnotetext{
* all inert primes about $3 \cdot 10^{4}$
} 


\begin{tabular}{|c|c|c|c|c|}
\hline$i$ & $\begin{array}{c}F_{A}=\text { size of the } \\
\text { algebraic factor base }\end{array}$ & $\begin{array}{c}\text { \# good pairs } \\
\text { of type } f f f\end{array}$ & $\begin{array}{c}\text { \# good pairs of } \\
\text { types } \text { pf } f \ldots p p p\end{array}$ & $\begin{array}{c}\text { \# cycles among } \\
\text { large primes }\end{array}$ \\
\hline \hline-27137 & 2537 & 6049 & 32154 & 18573 \\
\hline-27139 & 2532 & 5019 & 26906 & 13801 \\
\hline+23 & 2524 & 4811 & 27812 & 14665 \\
\hline+13 & 2492 & 4365 & 24790 & 12139 \\
\hline 0 & 2493 & 4390 & 24533 & 11931 \\
\hline-50467 & 2498 & 3552 & 21016 & 8985 \\
\hline+27140 & 2484 & 3354 & 19689 & 7843 \\
\hline-43467 & 2514 & 3240 & 18966 & 7499 \\
\hline-27142 & 2454 & 3181 & 18552 & 6998 \\
\hline+27138 & 2533 & 2797 & 16307 & 5407 \\
\hline
\end{tabular}

For the second table we modified the polynomial $f(x)$ obtained with $m=\left\lfloor n^{1 / 3}\right\rfloor$ by adding $\mathrm{g}(\mathrm{x})$.

\begin{tabular}{|c|c|c|c|c|}
\hline$g(x)$ & $\left|F_{A}\right|$ & $\begin{array}{l}\text { \# good pairs } \\
\text { of type } f f f\end{array}$ & $\begin{array}{l}\text { \# good pairs of } \\
\text { types } p f f \ldots p p p\end{array}$ & $\begin{array}{c}\text { \# cycles among } \\
\text { large primes }\end{array}$ \\
\hline \hline$-x^{2}+m x$ & 2535 & 6014 & 33224 & 20213 \\
\hline 0 & 2493 & 4390 & 24533 & 11931 \\
\hline$-x^{2}+(m+1) x-m$ & 2522 & 3245 & 18657 & 7204 \\
\hline$x-m$ & 2533 & 3080 & 16856 & 5620 \\
\hline$-2\left(x^{2}-(m-1) x-m\right)$ & 2348 & 1780 & 11312 & 2339 \\
\hline
\end{tabular}

\section{Some full factorizations}

The first numbers we factored with GNFS were

1. $n=68094773835969194533114212277$ using $f(x)=x^{3}+x^{2}-552450799 x+219569758, m=4083550467$

2. $n=8293575851234332290999689749600325042327$ using $f(x)=x^{3}+3011350157913 x+5946118091613, m=20241713503301$

3. $n=3488170797440166635069632321122160510282608893989$ using $f(x)=x^{3}+2 x^{2}+5137693962145733 x+2789637810783197$, $m=15165820588038497$

4. $n=9 \cdot 43622325302020166081169508345421120979479190926939307$ 2492793753701094144521495391401205652499957116372368586 1999536219765430952971290 using $f(x)=x^{5}+9, m=3^{58}$ 
All relations were found by Pollard's lattice sieve algorithm [7]. The most important datas of these factorizations are summarized in the following table.

\begin{tabular}{|c|c|c|c|c|}
\hline \# digits of $n$ & 29 & 40 & 49 & 134 \\
\hline \hline \multicolumn{4}{|c|}{ factor bases } \\
\hline $\begin{array}{c}\text { biggest prime of } \\
\text { the rational factor base }\end{array}$ & 5279 & 22307 & 30559 & 951161 \\
\hline size of the rational factor base & 700 & 2500 & 3300 & 75000 \\
\hline $\begin{array}{c}\text { bound for the large primes } \\
\text { on the rational side }\end{array}$ & $10^{5}$ & $6 \cdot 10^{5}$ & $10^{6}$ & $10^{8}$ \\
\hline $\begin{array}{c}\text { biggest prime p of the pairs (p,cp) } \\
\text { of the algebraic factor base }\end{array}$ & 22291 & 104729 & 224737 & 951109 \\
\hline size of the algebraic factor base & 2493 & 9794 & 19944 & 74952 \\
\hline $\begin{array}{c}\text { bound for the large prime } \\
\text { on the algebraic side }\end{array}$ & $10^{5}$ & $1.5 \cdot 10^{6}$ & $10^{7}$ & $10^{8}$ \\
\hline $\begin{array}{c}\text { \# additional pairs (p,cp) with } \\
\text { p bigger than large prime bound }\end{array}$ & 10 & 10 & 20 & 25 \\
\hline \hline
\end{tabular}

\section{finding the squares with the lattice sieve}

\begin{tabular}{|c|c|c|c|c|}
\hline sieving bound $C$ & 500 & 500 & 5000 & 10000 \\
\hline sieving bound $D$ & 50 & 200 & 1000 & 5250 \\
\hline \# good pairs of type $f f f$ & 4390 & 9133 & 8010 & 73798 \\
\hline \# good pairs of type $p f f$ & 4733 & 13020 & 16906 & 184864 \\
\hline \# good pairs of type $f p f$ & 6515 & 30937 & 46531 & 344560 \\
\hline \# good pairs of type $p p f$ & 8214 & 42681 & 109389 & 1031253 \\
\hline \# good pairs of type $f p p$ & 2272 & 17849 & 69304 & $0^{\star}$ \\
\hline \# good pairs of type $p p p$ & 2799 & 22862 & 153719 & $0^{\star}$ \\
\hline cycles among large primes & 11931 & 23386 & 19371 & 69103 \\
\hline sieving time in mips days & 1.75 & 118 & 717 & 41010 \\
\hline \multicolumn{5}{|c|}{ extracting the square root } \\
\hline \# inert primes & 150 & 175 & 240 & 105 \\
\hline size of inert primes about & $3 \cdot 10^{4}$ & $3 \cdot 10^{4}$ & $3 \cdot 10^{4}$ & $1.1 \cdot 10^{5}$ \\
\hline max. exponent for lifting & 16 & 64 & 64 & 256 \\
\hline \# digits of coefficients of the root & $\sim 8000$ & $\sim 51000$ & $\sim 69120^{\text {k太 }}$ & $\sim 135500^{\star \star}$ \\
\hline running time in mips hours & 7.5 & 36 & 41 & 484.5 \\
\hline
\end{tabular}

\footnotetext{
- only one large prime on each side

* with square reduction
} 
The factorizations are

1. 68094773835969194533114212277

$=17858990807069 \cdot 3812912754791033$

2. 8293575851234332290999689749600325042327

$=1301675260127398757 \cdot 6371463071690104808011$

3. 3488170797440166635069632321122160510282608893989

$=2203672182803847412085111 \cdot 1582890061715978295788099$

4. 4362232530202016608116950834542112097947919092693930724927 937537010941445214953914012056524999571163723685861999536219 765430952971290

$=2 \cdot 5 \cdot 557 \cdot 1107553 \cdot 8207398122145081$.

13857905391453292485606236633776204574597 .

621707356762164618894298788282728772085730542313277387634 1378217457

\section{References}

1. J. Buchmann, J. Loho, J. Zayer, An implementation of the general number field sieve, full version, to appear 1993

2. J. P. Buhler, H. W. Lenstra, C. Pomerance, Factoring integers with the number field sieve, Lecture Notes in Mathematics 1554, pp. 50 - 94. Springer Verlag, 1993

3. J. - M. Couveignes, Computing a square root for the number field sieve, Lecture Notes in Mathematics 1554, pp. 95 - 102, Springer Verlag, 1993

4. D. E. Knuth, The Art of Computer Programming, vol. 2, Second Edition, Addison Wesley, 1981

5. A. K. Lenstra, H. W. Lenstra, M. S. Manasse, J. M. Pollard, The number field sieve, Abstract: Proc. 22nd Ann. ACM Symp. on Theory of Computing (STOC)(1990),564-572

6. A. K. Lenstra, M. Manasse, Factoring with two large primes, preprint 1992

7. J. M. Pollard, The Lattice Sieve, Lecture Notes in Mathematics 1554, pp. 43 - 49, Springer Verlag, 1993

8. D. Shanks Five Number-Theoretic Algorithms, Proc. Second Manitoba Conference On Numerical Math., 1972, pp. 51-70 